\title{
Les sciences sociales françaises face à la gare. Bilan et lecture critique
}

French Social Science and railway stations. An evaluation and a critical reading

David Bàn

\section{(2) OpenEdition}

\section{Journals}

Édition électronique

URL : https://journals.openedition.org/rhcf/342

DOl : $10.4000 /$ rhcf.342

\section{Éditeur}

Rails \& histoire

Édition imprimée

Date de publication : 31 mai 2008

Pagination : 11-18

ISSN : 0996-9403

Référence électronique

David Bàn, «Les sciences sociales françaises face à la gare. Bilan et lecture critique », Revue d'histoire des chemins de fer [En ligne], 38 | 2008, mis en ligne le 10 mai 2011, consulté le 22 avril 2022. URL: http://journals.openedition.org/rhcf/342 ; DOI : https://doi.org/10.4000/rhcf.342 


\title{
Les sciences sociales françaises face à la gare. Bilan et lecture critique
}

\author{
David BÁN
}

ette présentation est un bref résumé de mon travail de
recherche achevé en 2005-2006, avec l'encouragement de
l'Association pour l'histoire des chemins de fer en France, et d'une partie de ma thèse de doctorat en sciences sociales, réalisée à l'École des hautes études en sciences sociales à Paris et à l'université Eötvös Loránd à Budapest sur les différentes approches socio-historiques de la gare. Ce travail concerne les exemples français, mais avec l'espoir d'une adaptation au cas de la Hongrie.

Avec l'essor urbain du XIX ${ }^{e}$ siècle, la gare est devenue un lieu multifonctionnel. Elle assure le passage de la ville à la campagne, du fermé à l'ouvert. Son statut de " porte de la ville » lui confère, encore au $\mathrm{XXI}^{\mathrm{e}}$ siècle, un rôle central dans l'organisation de l'espace tant d'un point de vue matériel que symbolique. Lieu public le plus récent de l'espace urbain, elle est riche d'enjeux architecturaux, industriels et historiques.

Mon étude, qui s'ancre dans le champ des sciences sociales, porte sur les usages sociaux de la gare urbaine comme espace public dans ses dimensions historique et architecturale, mais aussi, bien sûr, politique et économique. Il s'agit de comprendre comment les sciences sociales (au sens large, comme l'histoire, l'urbanisme, la sociologie, l'anthropologie) ont envisagé la gare d'un point de vue méthodologique comme épistémologique.

En France, les premières sources disponibles proviennent de l'histoire. Elles inscrivent la gare dans le double contexte de l'histoire des chemins de fer et de l'histoire économique. Dans les dernières décennies 
l'histoire s'est intéressée à la vie quotidienne, notamment dans l'espace urbain. En France, la structure de la ville moderne haussmannienne a été particulièrement étudiée, dans les relations qu'elle entretient avec le développement économique et industriel ainsi qu'avec le développement très remarquable des transports en commun.

Un deuxième champ d'investigation s'est ouvert autour de la question de l'emplacement des gares dans le tissu urbain. Ce champ est exploré par les urbanistes, qui replacent cette question de l'emplacement des gares dans la ville dans son contexte sociologique et économique et s'intéressent aussi bien à la gare qu'à son environnement.

La sociologie et l'anthropologie se sont également intéressées à la gare. Plus récentes que les précédentes, ces disciplines ne se sont en France tournées vers l'étude des problèmes spécifiquement urbains. Mais la question reste posée de la pertinence des méthodes propres à ces disciplines pour l'analyse des dimensions sociales et des usages d'un espace comme la gare.

Pour commencer cette analyse, j’ai recherché les différentes définitions historiques et morphologiques de la gare. Michel Ragon, spécialiste de l'architecture des gares, construit une approche linguistique en retenant la connotation du terme " garer » qui vient des transports fluviaux. Avec l'avènement du chemin de fer, la gare est ainsi devenue le centre, le « cœur» du réseau ferroviaire. Cependant, une fois cette présentation faite, Michel Ragon semble considérer la gare comme une évidence, comme un phénomène purement architectural, n'appelant du chercheur qu'un travail de description des bâtiments ${ }^{1}$.

Mais quel sens donner à la gare ? Quels cadres cognitifs lui appliquer? Faut-il y voir un type spécifique de bâtiment ? Un territoire industriel ? La perception commune, celle que véhicule le langage quotidien, semble à cet égard bien confuse.

Clive Lamming donne une définition très encyclopédique, plutôt technique de la gare, comme un « ensemble des installations de chemin de fer destinées, en un point déterminé, à permettre l'embarquement, le transbordement, la répartition ou le débarquement de voyageurs ou de marchandises $»^{2}$.

1- Michel Ragon, L'Architecture des gares. Naissance, apogée et déclin des gares de chemin de fer, Paris, Denoël, 1984, p. 9.

2- Clive Lamming, avec la collaboration de Daniel Brun, Pierre Cerisier et Alain Gernigon, Larousse des trains et des chemins de fer, Paris, Larousse, 2005, p. 283. 
Pour Karen Bowie également, la gare ne peut être réduite à sa seule dimension de «bâtiment voyageurs ». C'est une institution plus large, où se mêlent plusieurs professions, où sont installés des locaux techniques et différents types de bâtiments sur un territoire plus vaste (constructions autour des rails, dépôts, ateliers de maintenance, etc.) ${ }^{3}$.

L'histoire de la notion de la gare lui confère une signification particulièrement complexe. Avant l'apparition du chemin de fer, d'autres moyens de transports ont façonné son sens ; puis celui-ci s'est redéfini en interaction avec les transformations de la ville.

Mais, au-delà des différentes terminologies techniques ou historiques, la gare symbolise le déplacement et la vitesse. Avec les nouvelles inventions des architectes, en tant qu'institution ferroviaire la gare est bientôt devenue un symbole fort du progrès industriel, de la modernité et de l'économie et, en même temps, de l'urbanisme et du politique. Selon Jean Dethier, le commissaire général de l'exposition thématique "Le Temps des gares », organisée par le Centre Georges Pompidou à Paris en 1978, la gare a métamorphosé notre environnement et donc notre relation avec le milieu naturel, social et culturel ${ }^{4}$. Avec la vitesse, on peut réduire les distances et entretenir un nouveau rapport à l'espace et au temps. La gare, en tant que bâtiment public issu de la révolution industrielle, a transformé la société occidentale. L'architecture des gares cristallise la vie et la pensée politique du moment. La gare participe ainsi à la propagande idéologique de certains régimes, comme ce fut le cas en ex-Union Soviétique, en Italie avec la gare de Milan, emblème du fascisme, ou avec les projets de Speer dans le Berlin des années 1930.

Thème central de La Bête humaine chez Emile Zola, le chemin de fer et le mystérieux milieu de la gare sont également un sujet littéraire, cinématographique et, depuis Claude Monet, la gare est devenue une matière pour les artistes, qui y voient un signifiant de leur époque.

En dépit de l'ouverture de plus en plus grande de celle-ci aux phénomènes urbains, la gare est restée largement hors des cadres de l'analyse historique. Les premières études historiques sur la ville ont été menées dans la perspective d'une histoire politique : la chronique citadine y était mise en relation avec les événements de la vie politique nationale. Les sociétés urbaines ont ensuite fourni un terrain d'observation privilégié

3- Karen Bowie, "Introduction", in K. Bowie (dir.), Les Grandes Gares parisiennes au XIX ${ }^{e}$ siècle, Paris, Délégation à l'action artistique de la ville de Paris, s.d. [1987], p. 2326, citation p. 24.

4- Jean Dethier, Le Temps des gares, catal. expo., Paris, Centre national d'art et de culture Georges Pompidou, Centre de création industrielle, 1978, p. 8. 
lorsque, dans les années 1930, l'histoire économique et sociale s'est développée. À côté d'ouvrages explorant ces dimensions, la ville a également fait l'objet de recherches monographiques.

Toute histoire des chemins de fer commence par aborder la question de l'économie et celle du développement technique. Il est indispensable d'observer les relations entre l'État, les banquiers, les industriels, les idéologues, les techniciens et les porteurs de capitaux comme le soulignent Beatrice de Andia et Georges Ribeill ${ }^{5}$.

François Caron, professeur d'histoire économique et des techniques, a consacré sa thèse de doctorat d'État à l'histoire de la Compagnie du chemin de fer du Nord. Il est un des premiers chercheurs spécialisés dans l'histoire des chemins de fer en France. Selon lui, le fait que l'histoire des chemins de fer recoupe toujours un pan de l'histoire plus générale de sociétés est majeur ${ }^{6}$. En accord avec Karen Bowie ${ }^{7}$, François Caron estime que les spécialistes des sciences sociales ne doivent pas se focaliser uniquement sur les chemins de fer ou sur les gares, mais doivent au contraire replacer leurs analyses dans un contexte historique plus large. Si l'histoire doit observer la gare comme un lieu, « une scène » où se déploient des événements historiques, où quelque chose se passe, l'histoire de la gare considérée pour elle-même n'existe pas. La gare donne certes lieu à quelques événements ou faits saillants, mais ceux-ci ne sont qu'une part de l'histoire plus générale, et la gare n'est que le théâtre de ces faits.

Quand on fait la somme des différents points de vue et approches de la gare, il semble que la façon la plus évidente de la présenter est de passer par des travaux monographiques. Un des premiers ouvrages monographiques sur la gare est celui de René Clozier, historien et géographe, qui a soutenu sa thèse de doctorat sur la gare du Nord en $1940^{8}$.

Mais la bibliographie sur l'histoire des gares est relativement mince, ce qu'explique la complexité du sujet. La question des sources nécessaires pour mener ce type de recherche est tout particulièrement délicate à

5- Béatrice de Andia, « Entre les feux de l'État, de la ville et des compagnies 》, in Karen Bowie (dir.), Les Grandes Gares parisiennes..., op. cit., p. 8-22, p. 8 et Georges Ribeill, "Les fondains stratégiques des grandes gares parisiennes », ibid., 27-38, p. 30.

6- François Caron, Histoire de l'exploration d'un grand réseau. La Compagnie du chemin de fer du Nord, 1846-1937, Paris/La Haye, Mouton, 1973, p. 37-38.

7- Karen Bowie, "Avant-propos » in "Chemins de fer, architecture et ville», Revue d'histoire des chemins de fer, $\mathbf{n}^{\circ} 23$ (automne 2000), p. 5 .

8- René Clozier, La Gare du Nord, Paris, Librairie J.-B. Baillière et fils, 1940, p. 15. 
résoudre. D'une part, parce qu'on ne peut pas séparer l'étude de la gare de celle de l'ensemble du chemin de fer; d'autre part, en raison du caractère foncièrement pluridisciplinaire du sujet.

Il est presque impossible de séparer l'histoire des gares de l'histoire de l'architecture elle-même. Dans les premiers temps, l'évolution de la gare est celle d'un bâtiment totalement à inventer. La gare, en effet, n'a aucun précédent historique sur le plan fonctionnel ou architectural. C'est un domaine d'invention ex nihilo qui donne bientôt des résultats assez remarquables. L'histoire des gares est l'histoire de l'évolution d'un nouveau genre de bâtiment, doté de fonctions inédites. Dans leurs ouvrages sur l'architecture générale des gares, Michel Ragon puis Karen Bowie essaient de saisir des éléments particuliers de l'architecture, de construire une typologie des différentes sortes de gares, et d'analyser les fonctions et l'esthétique de ces bâtiments selon les périodes historiques.

Après l'histoire, l'urbanisme s'est tourné vers les sciences sociales pour penser la gare. L'emplacement de la gare dans le tissu urbain est certes avant tout un problème d'urbanisme mais ses conséquences sur le «visage » social de l'environnement ne peuvent être ignorées et la question des relations entre urbanisme et société doit être posée.

Les débats sur l'emplacement de la gare présentent une certaine constance. Au début de l'installation des chemins de fer au XIX siècle, la gare avait un rôle plutôt périphérique : elle était construite hors de la ville ou loin du centre. Cette conception semble redevenir d'actualité de nos jours, avec les nouvelles gares TGV en France, et partout dans le monde où l'on trouve des trains de la nouvelle génération. Les débats d'urbanisme opposent toujours d'un côté ceux pour qui la gare dynamise le quartier qui l'environne et, de l'autre, ceux pour qui elle le dégrade. Les nouveaux développements auxquels on assiste en France, et surtout l'idée de placer les gares hors de la ville, font l'objet de nombreuses critiques.

Après la Grande-Bretagne, la France a vu apparaitre des approches de la gare comme espace public. Les rôles sociaux très divers qui participent de son fonctionnement l'ont progressivement constituée en laboratoire pour les sciences sociales. Les sociologues et anthropologues se sont donné pour tâche de découvrir le fonctionnement interne de cette institution spécifiquement urbaine.

La nouveauté de ce terrain en fait un champ expérimental pour l'anthropologie et la sociologie qui ne disposent pas pour l'instant de méthodes convenables et établies pour ce type de recherche. Ces deux disciplines semblent toutefois indispensables à la compréhension de l'espace de la gare comme système. 
Isaac Joseph a essayé pour sa part de recomposer les différents éléments d'une théorie de la recherche urbaine à partir de l'École de Chicago, susceptible de déboucher sur un travail de terrain dans un univers comme la gare. Les méthodes de l'anthropologie et de la sociologie lui permettent d'analyser l'espace urbain, ses fonctions très complexes et l'économie des usages de ce territoire, comme le montrent quelques travaux de terrain. L'exemple peut-être le plus complexe d'aménagement d'un espace de gare est celui de la rénovation de la gare du Nord à Paris. Ce projet donne une idée des possibilités ouvertes en la matière par l'enquête de terrain et de l'importance de celle-ci dans les processus de rénovation.

À l'occasion de la mise en place, à la gare du Nord, d'une nouvelle ligne du TGV (le TGV Nord) ainsi que de nouvelles connections internationales (Eurostar et Thalys), a été mis sur pied un programme de recherche portant sur l'utilisation de l'espace public. À cette occasion, une équipe de recherche associant des sociologues, des architectes, des historiens, des historiens de l'art et des urbanistes a été constituée à la demande de la RATP et de la SNCF.

La gare, comme espace d'activité, d'interaction et de communication « interurbaine » pourrait être un terrain de référence pour la recherche urbaine, comme Isaac Joseph l'écrit :

« À partir de la gare, il s'agit donc de comprendre comment la ville peut modifier notre perception du social et de l'histoire sociale, d'analyser la construction de l'urbanité dans ses catégories propres et de retrouver la trace de l'expérience urbaine dans les enquêtes sur les compétences sociales des citadins?. »

9- Isaac Joseph, «Les compétences de rassemblement. Une ethnographie des lieux publics », in «La ville des sciences sociales », Enquête, 4 (1996), p. 107-122, p. 108. 


\section{Références bibliographiques (sélection)}

Agence des gares - Arep, Parcours 1988-1998 Paris-Rome, Agence des Gares-SNCF/AREP, Diagonale, 1998.

Berger, Eric, «Arras. La gare au centre de développement urbain 18462004 », in « Transports en ville », Histoire urbaine, no 11 (décembre 2004), p. 31-54.

Bowie, Karen (dir.), Les Grandes Gares parisiennes an XIX siècle, Paris, Délégation à l'action artistique de la ville de Paris, s.d. [1987].

Bowie, Karen, « De la gare du XIX ${ }^{\mathrm{e}}$ siècle au lieu-mouvement. Évolution ou rupture? ", in « Gares en mouvements ", Annales de la recherche urbaine, $\mathrm{n}^{\circ} 71$ (juin 1996), p. 14-23.

Bowie, Karen, "Avant-propos » in "Chemins de fer, architecture et ville », Revue d'histoire des chemins de fer, $\mathrm{n}^{\circ} 23$ (automne 2000).

Bowie, Karen ; Texier, Simon (dir.), Paris et ses chemins de fer, Paris, Action artistique de la ville de Paris, 2003.

Caron, François, Histoire de l'exploration d'un grand réseau. La Compagnie du chemin de fer du Nord, 1846-1937, Paris/La Haye, Mouton, 1973.

Caron, François, Les Grandes Compagnies de chemin de fer en France, 18231937, Genève, Droz, 2005.

Clozier, René, La Gare du Nord, Paris, Librairie J.-B. Baillière et fils, 1940.

Cognasson, Patrick, Gare de l'Est : porte ouverte sur l'Europe, Paris, La vie du rail, 1994.

Crosnier Leconte, Marie-Laure, La Naissance des gares, Paris, Hachette, 1990.

Dethier, Jean, Le Temps des gares, catal. expo., Paris, Centre national d'art et de culture Georges Pompidou, Centre de création industrielle, 1978.

Dethier, Jean, Gares d'Europe, Paris, Denoël, 1988.

Fillon, Odile ; Duthilleul, Jean-Marie ; Tricaud, Etienne, et alii, « Les nouvelles gares du TGV Méditerranée », Connaissance des arts, hors série $\mathrm{n}^{\circ} 172,2001$.

Heuraux, Christine, «La gare », in Krulic, Brigitte (dir.), Europe, lieux communs, Paris, Autrement, 2004, p. 163-178.

Joseph, Isaac, " Ariane et l'opportunisme méthodique » », in « Gares en mouvements », Annales de la recherche urbaine, no 71 (1996), p. 5-13.

Joseph, Isaac (dir.), Villes en gares, Paris, Éditions de l'Aube, 1999.

Joseph, Isaac, Gares intelligentes, accessibilité urbaine et relais de la ville dense, Paris, RATP, département du développement, mission prospective, 1999. 
Joseph, Isaac, «Les compétences de rassemblement. Une ethnographie des lieux publics », in « La ville des sciences sociales », Enquête, 4 (1996), p. 107-122.

Joseph, Isaac, Gare du Nord, mode d'emploi, Paris, Édition Recherches, 1997.

Lamming, Clive, Paris ferroviaire, Paris, Parigramme, 2002.

Lamming, Clive ; Marseille Jaques, Le Temps des chemins de fer en France, Paris, PML Editions, 1986.

Parissien, Steven, Station to Station, Londres-New York, Phaidon, 1997.

Ragon, Michel, L'Architecture des gares. Naissance, apogée et déclin des gares de chemin de fer, Paris, Denoël, 1984.

Ribeill, Georges, "Les métamorphoses de la grande gare française » », in « Gares en mouvements ", Annales de la recherche urbaine, $\mathrm{n}^{\circ} 71$ (1996), p. 54-65.

Roth, Ralf ; Polino, Marie-Noëlle (dir.), The City and the Railway in Europe, Aldershot-Burlington, Ashgate, 2003.

Wilson-Bareau, Juliet, Manet, Monet - La gare Saint-Lazare, Paris, Éditions de la Réunion des musées nationaux, 1998. 